\title{
Estimating unknown values in reciprocal intuitionistic preference relations via asymmetric fuzzy preference relations
}

\author{
Francisco Chiclana ${ }^{1}$, Raquel Ureña ${ }^{2}$, Hamido Fujita ${ }^{3}$, and Enrique \\ Herrera-Viedma ${ }^{2}$ \\ 1 Centre for Computational Intelligence, Faculty of Technology, De Montfort \\ University, Leicester, UK. chiclana@dmu.ac.uk \\ 2 Department of Computer Science and Artificial Intelligence, University of Granada, \\ Granada, Spain. viedma@decsai.ugr.es; raquel@decsai.ugr.es \\ 3 Iwate Prefectural University, Takizawa, Iwate, Japan, issam@iwate-pu.ac.jp
}

\begin{abstract}
Intuitionistic preference relations are becoming increasingly important in the field of group decision making since they present a flexible and simple way to the experts to provide their preference relations, while at the same time allowing them to accommodate a certain degree of hesitation inherent to all decision making processes. In this contribution, we prove the mathematical equivalence between the set of asymmetric fuzzy preference relations and the set of reciprocal intuitionistic fuzzy preference relations. This result is exploited to tackle the presence of incomplete reciprocal intuitionistic fuzzy preference relation in decision making by developing a consistency driven estimation procedure via the corresponding equivalent incomplete asymmetric fuzzy preference relation.
\end{abstract}

Keywords: Intuitionistic preference relation, asymmetric fuzzy preference relation, consistency, uninorm, incomplete information.

\section{Introduction}

Much research has been carried out in decision making with preferences modelled using fuzzy preference relations in comparison to using intuitionistic fuzzy preference relations. This is mainly to the longer existence of the former representation format of preferences but also to the increase computational complexity associated to the use of membership degree, non-membership degree and hesitation degree to model experts' subjective preferences with the latter representation format. Notice that in decision making, intuitionistic fuzzy preference relations are usually assumed to be reciprocal (Section 2).

In this paper the set of reciprocal intuitionistic fuzzy preference relations and the set of asymmetric fuzzy preference relations are proved to be mathematically isomorphic. The importance of this result resides in that it can be exploited to use methodologies developed for fuzzy preference relations to the case of intuitionistic fuzzy preference relations and, ultimately, to overcome the 
computation complexity mentioned above and to extend the use of reciprocal intuitionistic fuzzy preference relations in decision making. Indeed, this result will allow us to take advantage of mature and well defined methodologies developed for fuzzy preference relations in an intuitionistic context while at the same time taking advantage of the flexibility of reciprocal intuitionistic fuzzy preference relations to model vagueness/uncertainty. In particular, in this paper we illustrate how this isomorphic equivalence is used to address the presence of incomplete reciprocal intuitionistic fuzzy preference relations in decision making.

Incomplete information, as a result from the incapability of experts to provide complete information about their preferences, may happens more frequently than expected because experts do not have a precise or sufficient level of knowledge of part of the problem, lack of time, difficulty to distinguish up to which degree one preference is better than other, or due to the presence of conflicting alternatives, among others $[2,9]$. In the literature, different approaches to deal with missing or incomplete information for the case of using fuzzy preference relations as the representation format of preferences have been extensively studied [24]. Most of the existing approaches are based on a methodology that 'builds' the matrix driven by the concept of consistency of information [1-5, 10,12-14, 16, 17, 25].

The case of incomplete intuitionistic fuzzy preference relations has also been addressed in literature in $[26,25]$. In both cases, a methodology driven by consistency was also adopted, although the way consistency of reciprocal intuitionistic fuzzy preference relations was modelled was different. On the one hand, in [26] a straight forward transposition of the multiplicative consistency property for fuzzy preference relations was proposed for the case of reciprocal intuitionistic fuzzy preference relations, which later was proved to be incorrect [25] and publicly acknowledged by the authors that proposed it [27]. On the other hand, in [25] the concept of multiplicative consistency for reciprocal intuitionistic fuzzy preference relations was derived by formally extending the fuzzy preference relation multiplicative transitivity property via the use of both the extension principle [29] and representation theorem of fuzzy sets [28]. In this contribution, though, a different approach to incomplete reciprocal intuitionistic fuzzy preference relations is presented based on the aforementioned isomorphic mapping between the set of reciprocal intuitionistic fuzzy preference relations and the set of asymmetric fuzzy preference relations.

The rest of the paper is set out as follows: The first part presents the two mathematical frameworks for representing preferences (Section 2) and the basics concepts needed throughout the rest of paper (Section 3). The second part of the paper demonstrates the isomorphism between the set of reciprocal intuitionistic fuzzy preference relations and the set of asymmetric fuzzy preference relations (Section 4) and its use to present a methodology to estimate missing values of reciprocal intuitionistic fuzzy preference relations (Section 5). The final part of the paper includes conclusions drawn form the results obtained (Section 6). 


\section{Preference relations in decision making}

The comparison of two elements of a set of feasible alternatives $(X)$ by an expert can lead to the preference of one alternative to the other or to a state of indifference between them. Obviously, there is the possibility of an expert being unable to compare them. Two main mathematical models based on the concept of preference relation can be used in this context. In the first one, a preference relation is defined for each one of the above three possible preference states (preference, indifference, incomparability) [11], which is usually referred to as a preference structure on the set of alternatives [20]. The second one integrates the three possible preference states into a single preference relation [7]. In this paper, we focus on the second one as per the following definition:

Definition 1 (Preference Relation). A preference relation $P$ on a set $X$ is a binary relation $\mu_{P}: X \times X \longrightarrow D$, where $D$ is the domain of representation of preference degrees provided by the decision maker.

For a set $X$ of finite cardinality $(\# X=n)$ the following matrix representation of a preference relation $P$ is used: $P=\left(p_{i j}\right)$, with $p_{i j}=\mu_{P}\left(x_{i}, x_{j}\right)$ being interpreted as the degree or intensity of preference of alternative $x_{i}$ over $x_{j}$ $(i, j \in\{1,2, \ldots, n\})$. The elements of $P$ can be of a numeric or linguistic nature, i.e., could represent numeric or linguistic preferences, respectively [19]. The main types of numeric preference relations used in decision making are: crisp preference relations, additive preference relations, multiplicative preference relations, interval-valued preference relations and intuitionistic preference relations. In this contribution we focus on reciprocal intuitionistic fuzzy preference relations and their equivalence to a subclass of fuzzy preference relations, the asymmetric fuzzy preference relations.

\subsection{Fuzzy preference relation}

Recall that given a universal set $U$, with a generic element denoted by $x$, a fuzzy set $X$ in $U$ is a defined as a set of ordered pairs:

$$
X=\left\{\left(x, \mu_{X}(x)\right) \mid x \in U\right\}
$$

where $\mu_{X}: U \rightarrow[0,1]$ is called the membership function of $A$ and $\mu_{X}(x)$ represents the degree of membership of the element $x$ in $X$. In this context, the degree of non-membership of the element $x$ in $X$ is normally defined as $\nu_{X}(x)=$ $1-\mu_{X}(x)$, and as a consequence the following reciprocity property holds: $\mu_{X}(x)+$ $\nu_{X}(x)=1$. The reciprocal relationship between membership and non-membership makes the latter one unnecessary in the formulation as it can be derived from the former.

Definition 2 (Fuzzy Preference Relation). A fuzzy preference relation $R=$ $\left(r_{i j}\right)$ on a finite set of alternatives $X$ is a relation in $X \times X$ that is characterised by a membership function $\mu_{R}: X \times X \longrightarrow[0,1]$. 
The following interpretation is assumed:

- $r_{i j}=1$ indicates the maximum degree of preference for $x_{i}$ over $x_{j}$

- $\left.r_{i j} \in\right] 0.5,1\left[\right.$ indicates a definite preference for $x_{i}$ over $x_{j}$

- $r_{i j}=1 / 2$ indicates indifference between $x_{i}$ and $x_{j}$

When $r_{i j}+r_{j i}=1(\forall i, j \in\{1, \ldots, n\})$ is imposed we have a reciprocal fuzzy preference relation.

\subsection{Intuitionistic fuzzy preference relation}

An intuitionistic fuzzy set $X$ over a universe of discourse $U$ is represented as [6]

$$
X=\left\{\left(x,\left\langle\mu_{X}(x), \nu_{X}(x)\right\rangle\right) \mid x \in U\right\}
$$

where $\mu_{X}: U \longrightarrow[0,1]$ and $\nu_{X}: U \longrightarrow[0,1]$ verify

$$
0 \leq \mu_{X}(x)+\nu_{X}(x) \leq 1 \quad \forall x \in U .
$$

In this context, $\mu_{X}(x)$ and $\nu_{X}(x)$ are known as the degree of membership and degree of non-membership of $x$ to $X$. Obviously, an intuitionistic fuzzy set becomes a fuzzy set when $\mu_{X}(x)=1-\nu_{X}(x) \forall x \in U$. However, when there exists at least a value $x \in U$ for which $\mu_{X}(x)<1-\nu_{X}(x)$, an extra parameter known as the hesitancy degree is defined with intuitionistic fuzzy sets, $\tau_{X}(x)=1-\mu_{X}(x)-\nu_{X}(x)$, representing the amount of lacking information in determining the membership of $x$ to $X$.

In [22], Szmidt and Kacprzyk defined the intuitionistic fuzzy preference relation as a generalisation of the concept of fuzzy preference relation.

Definition 3 (Intuitionistic Fuzzy Preference Relation). An intuitionistic fuzzy preference relation $B$ on a finite set of alternatives $X=\left\{x_{1}, \ldots, x_{n}\right\}$ is characterised by a membership function $\mu_{B}: X \times X \rightarrow[0,1]$ and a nonmembership function $\nu_{B}: X \times X \rightarrow[0,1]$ such that

$$
0 \leq \mu_{B}\left(x_{i}, x_{j}\right)+\nu_{B}\left(x_{i}, x_{j}\right) \leq 1 \quad \forall\left(x_{i}, x_{j}\right) \in X \times X .
$$

The value $\mu_{B}\left(x_{i}, x_{j}\right)=\mu_{i j}$ is interpreted as the certainty degree up to which $x_{i}$ is preferred to $x_{j}$, while $\nu_{B}\left(x_{i}, x_{j}\right)=\nu_{i j}$ is interpreted as the certainty degree up to which $x_{i}$ is non-preferred to $x_{j}$.

As with a fuzzy preference relation, an intuitionistic fuzzy preference relation is represented by a matrix $B=\left(b_{i j}\right)$ with $b_{i j}=\left\langle\mu_{i j}, \nu_{i j}\right\rangle \forall i, j=1,2, \ldots, n$. Obviously, when the hesitancy function is the null function we have that $\mu_{i j}+\nu_{i j}=$ $1(\forall i, j)$ and the intuitionistic fuzzy preference relation $B=\left(b_{i j}\right)$ is mathematically equivalent to the reciprocal fuzzy preference relation $R=\left(r_{i j}\right)$, with $r_{i j}=\mu_{i j}$.

An intuitionistic fuzzy preference relation is referred to as reciprocal when the following additional conditions are imposed:

$$
\begin{aligned}
& -\mu_{i i}=\nu_{i i}=0.5 \forall i \in\{1, \ldots, n\} . \\
& -\mu_{j i}=\nu_{i j} \forall i, j \in\{1, \ldots, n\} .
\end{aligned}
$$




\section{Consistency of fuzzy preferences}

Consistency of fuzzy preference relations has been modelled using the notion of transitivity in the pairwise comparison among any three alternatives. If $x_{i}$ is preferred to $x_{j}\left(x_{i} \succ x_{j}\right)$ and this one to $x_{k}\left(x_{j} \succ x_{k}\right)$ then alternative $x_{i}$ should be preferred to $x_{k}\left(x_{i} \succ x_{k}\right)$, which is normally referred to as weak stochastic transitivity [18]. Any property that guarantees the transitivity of the preferences is called a consistency property [8].

Different properties or conditions have been suggested as rational to be verified by a consistent fuzzy preference relation $[8,15]$ : triangle condition, weak transitivity, max-min transitivity, max-max transitivity, restricted max-min transitivity, restricted max-max transitivity, additive transitivity, and multiplicative transitivity. The last two properties, proposed by Tanino in [23], are the most widely used in the context of incomplete information [8].

Definition 4 (Additive transitivity). A fuzzy preference relation $R=\left(r_{i j}\right)$ on a finite set of alternatives $X$ is additive transitive if and only if

$$
\left(r_{i j}-0.5\right)+\left(r_{j k}-0.5\right)=r_{i k}-0.5 \quad \forall i, j, k=1,2, \cdots, n
$$

Additive transitivity for fuzzy preference relations is equivalent to Saaty's consistency property [21] for multiplicative preference relations [15]. However, it is also a fact that additive transitivity is in conflict with the $[0,1]$ scale used for providing the preference values and therefore it is not appropriate to model consistency of fuzzy preference relations [8]. An alternative transitivity property for fuzzy preference relations to additive transitivity was also proposed by Tanino [23]:

Definition 5 (Multiplicative transitivity). A fuzzy preference relation $R=$ $\left(r_{i j}\right)$ on a finite set of alternatives $X$ is multiplicative transitive if and only if

$$
r_{i j} \cdot r_{j k} \cdot r_{k i}=r_{i k} \cdot r_{k j} \cdot r_{j i} \forall i, k, j \in\{1,2, \ldots n\}
$$

Multiplicative transitivity extends weak stochastic transitivity, and therefore extends the classical transitivity property of crisp preference relations.

The modelling of cardinal consistency of reciprocal fuzzy preference relations via a functional equation was proposed in [8], and it was proved that when such a function is almost continuous and monotonic (increasing) then it must be a representable uninorm. Furthermore, cardinal consistency with the conjunctive representable cross ratio uninorm

$$
U(x, y)= \begin{cases}0, & (x, y) \in\{(0,1),(1,0)\} \\ \frac{x y}{x y+(1-x)(1-y)}, & \text { otherwise }\end{cases}
$$

is equivalent to Tanino's multiplicative transitivity property as per Definition 5. As any two representable uninorms are order isomorphic, it was concluded that multiplicative transitivity is the most appropriate property to model consistency 
of reciprocal fuzzy preference relations. This property is referred though as the multiplicative consistency property.

Multiplicative consistency property (1) allows to estimate the (fuzzy) preference value between a pair of alternatives $\left(x_{i}, x_{j}\right)$ with $(i<j)$ using a different intermediate alternative $x_{k}(k \neq i, j), m r_{i j}^{k}$, as

$$
m r_{i j}^{k}=\frac{r_{i k} \cdot r_{k j} \cdot r_{j i}}{r_{j k} \cdot r_{k i}}
$$

so long as the denominator is not zero. The value $m r_{i j}^{k}$ is known as the partially multiplicative transitivity based estimated fuzzy preference value of the pair of alternatives $\left(x_{i}, x_{j}\right)$ obtained using the intermediate alternative $x_{k}$.

The following points are noted:

- Expression (1) is always true when two of the three subindexes in $\{i, j, k\}$ are equal.

- When $k=i$ and $r_{j i} \neq 0$ then $m r_{i j}^{i}=r_{i j}$, while when $r_{i j} \neq 0$ then $m r_{j i}^{i}=r_{j i}$. Because $r_{j i}=1-r_{i j}$, then we have that: $r_{j i} \neq 0$ if and only if $r_{j i} \neq 1$. Thus, if $k=i$ and $\left(r_{i j}, r_{j i}\right) \notin\{(1,0),(0,1)\}$ we have $m r_{i j}^{i}=r_{i j}$ and $m r_{j i}^{i}=r_{j i}$.

- A similar reasoning and conclusion is obtained when $k=j$.

- Although it is possible to obtain the multiplicative transitivity based estimated fuzzy preference value of the pair of alternatives $\left(x_{i}, x_{j}\right)$ when $k \in$ $\{i, j\}$ and $\left(r_{i j}, r_{j i}\right) \notin\{(1,0),(0,1)\}$, it is also true that there is no indirect estimation process as described above.

- When the fuzzy preference value $r_{i j}$ is unknown its estimation will automatically require that $k \notin\{i, j\}$.

- Finally, when $i=j$ we have by definition that $r_{i i}=0.5$ and we would have $m r_{i i}^{k}=r_{i i}$ whenever $r_{i k} \notin\{(0,1),(1,0)\}$. Thus, this case will not be relevant when having incomplete information.

Thus, the global multiplicative transitivity based estimated value of the fuzzy preference value of the pair of alternatives $\left(x_{i}, x_{j}\right)$ is defined as the following average of partially multiplicative transitivity based estimated values

$$
m r_{i j}=\frac{\sum_{k \in R_{i j}^{01}} m r_{i j}^{k}}{\# R_{i j}^{01}} ;
$$

where $R_{i j}^{01}=\left\{k \neq i, j \mid\left(r_{i k}, r_{k j}\right) \notin R^{01}\right\}, R^{01}=\{(1,0),(0,1)\}$, and $\# R_{i j}^{01}$ is the cardinality of $R_{i j}^{01}$.

Given a fuzzy preference relation, $R=\left(r_{i j}\right)$, its multiplicative transitivity based fuzzy preference relation, $M R=\left(m r_{i j}\right)$, can be constructed. If $R$ is multiplicative transitive then (1) holds $\forall i, j, k$, and we have

$$
r_{i j}=\frac{r_{i k} \cdot r_{k j} \cdot r_{j i}}{r_{j k} \cdot r_{k i}}
$$

whenever $k \in R_{i j}^{01}$. Consequently, $m r_{i j}^{k}=r_{i j} \forall i, j, k \in R_{i j}^{01}$ and therefore it is $r_{i j}=m r_{i j} \forall i, j$. The following alternative definition of multiplicative transitivity for fuzzy preference relations is justified. 
Definition 6 (Multiplicative Consistency). A fuzzy preference relation $R=$ $\left(r_{i j}\right)$ is multiplicative consistent if and only if $R=M R$.

The similarity value between the fuzzy preference relation, $R$, and its its multiplicative transitivity based fuzzy preference relation, $M R$ is defined as follows:

$$
C L=\frac{\sum_{i, j=1 ; i \neq j}^{n} C L_{i j}}{n \cdot(n-1)} .
$$

where $C L_{i j}=1-d\left(r_{i j}, m r_{i j}\right) \quad \forall i, j$, and $d\left(r_{i j}, m r_{i j}\right)$ represents the distance between the values $r_{i j}$ and $m r_{i j}$. We have the following:

- If $R$ is multiplicative consistent then it is $r_{i j}=m r_{i j} \forall i, j$. Consequently, $d\left(r_{i j}, m r_{i j}\right)=0 \forall i, j$, i.e. $C L=1$.

- If $C L=1$ then $2 \cdot \sum_{i, j=1 ; i<j}^{n} C L_{i j}=n \cdot(n-1)$. Commutativity of $d$ implies that $C L_{i j}=C L_{j i} \forall i, j$. Therefore it is $\sum_{i, j=1, i \neq j}^{n} C L_{i j}=n \times(n-1)$, and because $C L_{i j} \in[0,1]$ then $C L_{i j}=1 \forall i \neq j$, i.e. $r_{i j}=m r_{i j} \forall i \neq j$. Finally, when $i=j$ we have $m r_{i i}^{k}=r_{i i}=0.5$ whenever $r_{i k} \notin\{(0,1),(1,0)\}$, and therefore $m r_{i i}=0.5 \forall i$. Thus, we have $r_{i j}=m r_{i j} \forall i, j$, i.e. $R=M R$, and $R$ is multiplicative consistent.

This proves that a fuzzy preference relation $R$ is multiplicative consistent if and only if $C L=1$, and therefore provides a characterisation of multiplicative consistency of a fuzzy preference relation based on its similarity value to its multiplicative transitivity based fuzzy preference relation.

\section{Reciprocal intuitionistic fuzzy preference relations and asymmetric fuzzy preference relations}

Let denote with $\mathcal{B}$ the set of reciprocal intuitionistic fuzzy preference relations:

$$
\begin{aligned}
\mathcal{B}=\left\{B=\left(b_{i j}\right) \mid \forall i j: b_{i j}\right. & =\left\langle\mu_{i j}, \nu_{i j}\right\rangle, \mu_{i j}, \nu_{i j} \in[0,1], \\
\mu_{i i} & \left.=\nu_{i i}=0.5 \mu_{i j}=\nu_{j i}, 0 \leq \mu_{i j}+\nu_{i j} \leq 1\right\}
\end{aligned}
$$

and with $\mathcal{R}$ the set of fuzzy preference relations

$$
\mathcal{R}=\left\{R=\left(r_{i j}\right) \mid \forall i j: r_{i j} \in[0,1]\right\}
$$

Let $f:[0,1] \times[0,1] \longrightarrow[0,1]$ be the following function $f\left(x_{1}, x_{2}\right)=x_{1}$. We can define the following mapping, $F: \mathcal{B} \longrightarrow \mathcal{R}$, between the set of reciprocal intuitionistic fuzzy preference relations, $\mathcal{B}$, and the set of fuzzy preference relations, $\mathcal{R}$,

$$
R=F(B)=\left(f\left(b_{i j}\right)\right)=\left(\mu_{i j}\right) .
$$

We have: 
- Function $F$ is well defined, i.e. given $B \in \mathcal{B}$ it is true that $f(B) \in \mathcal{R}$.

- Function $F$ is an injection. Indeed, let $B_{1}=\left(b_{i j}^{1}\right)$ and $B_{2}=\left(b_{i j}^{2}\right)$ two reciprocal intuitionistic fuzzy preference relation such that $F\left(B_{1}\right)=F\left(B_{2}\right)$. Then we have that

$$
f\left(b_{i j}^{1}\right)=f\left(b_{i j}^{2}\right) \Leftrightarrow \mu_{i j}^{1}=\mu_{i j}^{2} \forall i, j .
$$

Because $\mu_{i j}^{1}=\nu_{j i}^{1}$ and $\mu_{i j}^{2}=\nu_{j i}^{2}$ then it is obvious that

Therefore we have that

$$
\nu_{i j}^{1}=\nu_{i j}^{2} \forall i, j .
$$

$$
b_{i j}^{1}=\left\langle\mu_{i j}^{1}, \nu_{i j}^{1}\right\rangle=\left\langle\mu_{i j}^{2}, \nu_{i j}^{2}\right\rangle=b_{i j}^{2} \forall i, j .
$$

Consequently, it is concluded that

$$
B_{1}=B_{2} .
$$

- Function $F$ is not a surjection as not all fuzzy preference relations $R \in \mathcal{R}$ verify $0 \leq r_{i j}+r_{j i} \leq 1$. Thus the range of function function $f$ is the set of asymmetric fuzzy preference relations.

Summarising:

The set of reciprocal intuitionistic fuzzy preference relations is isomorphic to the set of asymmetric fuzzy preference relations.

\section{Estimating unknown values in incomplete reciprocal intuitionistic fuzzy preference relations}

It is assumed that for incomplete reciprocal intuitionistic fuzzy preference relations, given a pair of alternatives $\left(x_{i}, x_{j}\right)$ for which $b_{i j}$ is not known, both membership and non-memberships will be unknown. Due to reciprocity, we have that if $b_{i j}$ is not known then $b_{j i}$ is also not known.

If $B$ is an incomplete reciprocal intuitionistic fuzzy preference relation, then $R=F(B)$ will be an incomplete asymmetric fuzzy preference relation. However, the missing preference value $r_{i j}(i \neq j)$ cannot be partially estimated, using an intermediate alternative $x_{k}$, via expression (1) because $r_{i j}$ is also unknown. In these cases we use expression (2). Thus the missing preference value $r_{i j}(i \neq j)$ can be partially estimated, using an intermediate alternative $x_{k}$, with the value:

$$
c r_{i j}^{k}= \begin{cases}0, & \left(r_{i k}, r_{k j}\right) \in\{(0,1),(1,0)\} \\ \frac{r_{i k} \cdot r_{k j}}{r_{i k} \cdot r_{k j}+\left(1-r_{i k}\right) \cdot\left(1-r_{k j}\right)}, & \text { Otherwise. }\end{cases}
$$

The global multiplicative transitivity based estimated value, $c r_{i j}$, is defined as:

$$
c r_{i j}=\frac{\sum_{k \in R_{i j}^{01}} c r_{i j}^{k}}{\# R_{i j}^{01}}
$$


where $H_{i j}^{01}=\left\{k \in R_{i j}^{01} \mid(i, j) \in M V \&(i, k),(k, j) \in E V\right\} ; M V$ is the set of pairs of different alternatives for which the fuzzy preference degree is unknown or missing; $E V$ is the set of pairs of different alternatives with known fuzzy preference values.

The iterative procedure to complete reciprocal fuzzy preference relations developed in [14] can be applied to complete $R$ and, consequently, to complete $B$ as the following example illustrates.

Example 1. Let $X=\left\{x_{1}, x_{2}, x_{3}, x_{4}\right\}$ be a set of alternatives evaluated by a decision maker against a particular criterion using the following incomplete reciprocal intuitionistic fuzzy preference relation [25]:

$$
B=\left(\begin{array}{cccc}
\langle 0.50,0.50\rangle & \langle 0.40,0.30\rangle & x & x \\
\langle 0.30,0.40\rangle & \langle 0.50,0.50\rangle & \langle 0.50,0.40\rangle & x \\
x & \langle 0.40,0.50\rangle & \langle 0.50,0.50\rangle & \langle 0.30,0.40\rangle \\
x & x & \langle 0.40,0.30\rangle & \langle 0.50,0.50\rangle
\end{array}\right)
$$

The associated incomplete asymmetric fuzzy preference relation is:

$$
R=\left(\begin{array}{cccc}
0.5 & 0.4 & - & - \\
0.3 & 0.5 & 0.5 & - \\
- & 0.4 & 0.5 & 0.3 \\
- & - & 0.4 & 0.5
\end{array}\right)
$$

Step 1: The set of elements that can be estimated at this stage are:

$$
E M V_{1}=\{(1,3),(2,4),(3,1),(4,2)\}
$$

The computation of the estimated values $c r_{13}$ and $c r_{31}$ requires the intermediate alternative $k=2$, for which we have

$$
c r_{13}^{2}=\frac{r_{12} \cdot r_{23}}{r_{12} \cdot r_{23}+\left(1-r_{12}\right) \cdot\left(1-r_{23}\right)}=\frac{0.4 \cdot 0.5}{0.4 \cdot 0.5+0.6 \cdot 0.5}=0.4 \text {, }
$$

and

$$
c r_{31}^{2}=\frac{r_{32} \cdot r_{21}}{r_{32} \cdot r_{21}+\left(1-r_{32}\right) \cdot\left(1-r_{21}\right)}=\frac{0.4 \cdot 0.3}{0.4 \cdot 0.3+0.6 \cdot 0.7}=0.22
$$

The computation of the estimated values $c r_{24}$ and $c r_{42}$ is done using intermediate alternative $k=3$

$$
c r_{24}^{3}=\frac{r_{23} \cdot r_{34}}{r_{23} \cdot r_{34}+\left(1-r_{23}\right) \cdot\left(1-r_{34}\right)}=\frac{0.5 \cdot 0.3}{0.5 \cdot 0.3+0.5 \cdot 0.7}=0.3,
$$

and

$$
c r_{42}^{3}=\frac{r_{43} \cdot r_{32}}{r_{43} \cdot r_{32}+\left(1-r_{43}\right) \cdot\left(1-r_{32}\right)}=\frac{0.4 \cdot 0.4}{0.4 \cdot 0.4+0.6 \cdot 0.6}=0.31
$$


After the estimation process is applied, we have:

$$
R=\left(\begin{array}{cccc}
0.5 & 0.4 & \mathbf{0 . 4} & - \\
0.3 & 0.5 & 0.5 & \mathbf{0 . 3} \\
\mathbf{0 . 2 2} & 0.4 & 0.5 & 0.3 \\
- & \mathbf{0 . 3 1} & 0.4 & 0.5
\end{array}\right)
$$

Step 2: The remaining unknown elements can be estimated at this stage, $E M V_{2}=\{(1,4),(4,1)\}$. The computation process of the estimated values are:

$$
\begin{gathered}
c r_{14}^{2}=\frac{r_{12} \cdot r_{24}}{r_{12} \cdot c_{24}+\left(1-c_{12}\right) \cdot\left(1-c_{24}\right)}=\frac{0.4 \cdot 0.3}{0.4 \cdot 0.3+0.6 \cdot 0.7}=0.22 \\
c r_{14}^{3}=\frac{r_{13} \cdot r_{34}}{r_{13} \cdot r_{34}-\left(1-r_{13}\right) \cdot\left(1-r_{34}\right)}=\frac{0.4 \cdot 0.3}{0.4 \cdot 0.3+0.6 \cdot 0.7}=0.22 \\
c r_{14}=\frac{c r_{14}^{2}+c r_{14}^{3}}{2}=0.22 . \\
c r_{41}^{2}=\frac{r_{42} \cdot r_{21}}{r_{42} \cdot c_{21}+\left(1-c_{42}\right) \cdot\left(1-c_{21}\right)}=\frac{0.31 \cdot 0.3}{0.31 \cdot 0.3+0.69 \cdot 0.7}=0.16 \\
r_{41}^{3}=\frac{r_{43} \cdot r_{31}}{r_{43} \cdot r_{31}-\left(1-r_{43}\right) \cdot\left(1-r_{31}\right)}=\frac{0.4 \cdot 0.22}{0.4 \cdot 0.22+0.6 \cdot 0.78}=0.16 \\
c r_{41}=\frac{c r_{41}^{2}+c r_{41}^{3}}{2}=0.16
\end{gathered}
$$

The following completed asymmetric fuzzy preference relation $R$ is obtained:

$$
R=\left(\begin{array}{cccc}
0.5 & 0.4 & \mathbf{0 . 4} & \mathbf{0 . 2 2} \\
0.3 & 0.5 & 0.5 & \mathbf{0 . 3} \\
\mathbf{0 . 2 2} & 0.4 & 0.5 & 0.3 \\
\mathbf{0 . 1 6} & \mathbf{0 . 3 1} & 0.4 & 0.5
\end{array}\right)
$$

The complete reciprocal intuitionistic fuzzy preference relation is:

$$
B=F^{-1}(R)=\left(\begin{array}{cccc}
\langle 0.50,0.50\rangle & \langle 0.40,0.30\rangle & \langle\mathbf{0 . 4 0 , 0 . 2 2}\rangle & \langle\mathbf{0 . 2 2}, \mathbf{0 . 1 6}\rangle \\
\langle 0.30,0.40\rangle & \langle 0.50,0.50\rangle & \langle 0.50,0.40\rangle & \langle\mathbf{0 . 3 0 , 0 . 3 1}\rangle \\
\langle\mathbf{0 . 2 2}, \mathbf{0 . 4 0}\rangle & \langle 0.40,0.50\rangle & \langle 0.50,0.50\rangle & \langle 0.30,0.40\rangle \\
\langle\mathbf{0 . 1 6}, \mathbf{0 . 2 2}\rangle & \langle\mathbf{0 . 3 1}, \mathbf{0 . 3 0}\rangle & \langle 0.40,0.30\rangle & \langle 0.50,0.50\rangle
\end{array}\right)
$$

Notice that the completed reciprocal intuitionistic fuzzy preference relation obtained coincides with the one in [25], where there was a typo in $b_{41}\left(b_{14}\right)$ that appeared as $\langle 0.19,0.22\rangle(\langle 0.22,0.19\rangle)$ instead of the correct one shown here. 


\section{Conclusion}

The set of asymmetric fuzzy preference relations is isomorphic to the set of reciprocal intuitionistic fuzzy preference relations. This result is important because it allows to use methodologies developed for fuzzy preference relations to the case of intuitionistic fuzzy preference relations and, ultimately, to overcome their associated computation complexity and ultimately to extend the use of reciprocal intuitionistic fuzzy preference relations in decision making. Indeed, this result has been exploited here to address the issue of incomplete reciprocal intuitionistic fuzzy preference relations in decision making.

\section{Acknowledgments}

This work has been developed with the financing of the Andalusian Excellence research project TIC-5991 and FEDER funds in the Spanish National research project TIN2013-40658-P. Raquel Ureña would like to acknowledge the support received by the mobility grant program awarded by the University of Granada 's International Office. Prof. Francisco Chiclana and Prof. Hamido Fujita would like to acknowledge the support provided by the University of Granada 'Strengthening through Short-Visits' (Ref. GENIL-SSV 2015) programme.

\section{References}

1. Alonso, S., Cabrerizo, F., Chiclana, F., Herrera, F., Herrera-Viedma, E., 2009a. Group decision making with incomplete fuzzy linguistic preference relations. Int. J. Intell. Syst 24 (2), 201-222.

2. Alonso, S., Chiclana, F., Herrera, F., Herrera-Viedma, E., 2004. A learning procedure to estimate missing values in fuzzy preference relations based on additive consistency. Lecture Notes in Artificial Intelligence 3131, 227-238.

3. Alonso, S., Chiclana, F., Herrera, F., Herrera-Viedma, E., Alcalá-Fdez, J., Porcel, C., 2008. A consistency-based procedure to estimate missing pairwise preference values. International Journal of Intelligent Systems 23 (2), 155-175.

4. Alonso, S., Herrera-Viedma, E., Chiclana, F., Herrera, F., 2009b. Individual and social strategies to deal with ignorance situations in multi-person decision making. International Journal of Information Technology and decision Making 8 (2), 313333.

5. Alonso, S., Herrera-Viedma, E., Chiclana, F., Herrera, F., 2010. A web based consensus support system for group decision making problems and incomplete preferences. Information Sciences 180 (23), 4477-4495.

6. Atanassov, K. T., 1986. Intuitionistic fuzzy sets. Fuzzy Sets and Systems 20 (1), 87-96.

7. Bezdek, J., Spillman, B., R., R. S., 1978. A fuzzy relation space for group decision theory. Fuzzy Sets and Systems 1 (4), 255-268.

8. Chiclana, F., Herrera-Viedma, E., Alonso, S., Herrera, F., 2009. Cardinal consistency of reciprocal preference relations: A characterization of multiplicative transitivity. IEEE Transactions on Fuzzy Systems 17 (1), 14-23. 
9. Ebenbach, D. H., Moore, C., 2000. Incomplete information, inferences, and individual differences: The case of environmental judgments. Organizational Behavior and Human Decision Processes 81 (1), 1-27.

10. Fedrizzi, M., Giove, S., 2007. Incomplete pairwise comparison and consistency optimization. European Journal of Operational Research 183 (1), 303-313.

11. Fishburn, P., 1979. Utility theory for decision making. Krieger, Melbourne, FL.

12. Genc, S., Boran, F. E., Akay, D., Xu, Z., 2010. Interval multiplicative transitivity for consistency, missing values and priority weights of interval fuzzy preference relations. Information Sciences 180 (24), 4877 - 4891.

13. Herrera-Viedma, E., Alonso, S., Chiclana, F., F.Herrera, 2007a. A consensus model for group decision making with incomplete fuzzy preference relations. IEEE Transactions on Fuzzy Systems 15 (5), 863-877.

14. Herrera-Viedma, E., Chiclana, F., F.Herrera, Alonso, S., 2007b. Group decisionmaking model with incomplete fuzzy preference relations based on additive consistency. IEEE Transactions on Systems, Man, and Cybernetics, Part B: Cybernetics 37 (1), 176-189.

15. Herrera-Viedma, E., Herrera, F., Chiclana, F., Luque, M., 2004. Some issues on consistency of fuzzy preference relations. European Journal of Operational Research 154 (1), 98-109.

16. Lee, L.-W., 2012. Group decision making with incomplete fuzzy preference relations based on the additive consistency and the order consistency. Expert Systems with Applications 39 (14), 11666-11676.

17. Liu, X., Pan, Y., Xu, Y., Yu, S., 2012. Least square completion and inconsistency repair methods for additively consistent fuzzy preference relations. Fuzzy Sets and Systems 198 (1), 1-19.

18. Luce, R. D., Suppes, P., 1965. Preferences, utility and subject probability. Handbook of mathematical psychology. Vol. 3. New York.

19. Pérez-Asurmendi, P., Chiclana, F., 2014. Linguistic majorities with difference in support. Applied Soft Computing 18, 196 - 208.

20. Roubens, M., Vincke, P., 1985. Preference modeling. Springer, Berlin.

21. Saaty, T. L., 1980. The analytic hierarchy process. McGraw-Hill.

22. Szmidt, E., Kacprzyk, J., 2002. Using intuitionistic fuzzy sets in group decision making. Control and Cybernetics 31 (4), 1037-1053.

23. Tanino, T., 1984. Fuzzy preference orderings in group decision making. Fuzzy sets and system $12,117-131$

24. Ureña, R., Chiclana, F., Morente-Molinera, J., Herrera-Viedma, E., 2015. Managing incomplete preference relations in decision making: A review and future trends. Information Sciences 302 (0), $14-32$.

25. Wu, J., Chiclana, F., 2014. Multiplicative consistency of intuitionistic reciprocal preference relations and its application to missing values estimation and consensus building. Knowledge-Based Systems 71 (0), 187 - 200.

26. Xu, Z., Cai, X., Szmidt, E., 2011. Algorithms for estimating missing elements of incomplete intuitionistic preference relations. International Journal of Intelligent Systems 26 (9), 787-813.

27. $\mathrm{Xu}, \mathrm{Z}$, Liao, H, 2014. A survey of approaches to decision making with intuitionistic fuzzy preference relations. Knowledge-based systems, 2015, doi: 10.1016/j.knosys.2014.12.034 (in press)

28. Zadeh, L. A., 1965. Fuzzy sets. Information and Control 8 (3), 338-357.

29. Zadeh, L. A., 1975. The concept of a linguistic variable and its application to approximate reasoning-i. Information Sciences 8, 199-249. 